\title{
Enzyme engineering by random mutagenesis: structural and functional studies of modified plant-type L-asparaginase (EcAllI)
}

\author{
J.I. Loch ${ }^{1}$, A. Klonecka1 , K. Kądziołka1, P. Bonarek², J. Barciszewski ${ }^{3}$, K. Lewiński ${ }^{1}$, M. Jaskolski ${ }^{3,4}$ \\ ${ }^{1}$ Jagiellonian University, Faculty of Chemistry, Krakow, Poland, ${ }^{2}$ Jagiellonian University, Faculty of Biochemistry, Biophysics and \\ Biotechnology, Krakow, Poland, ${ }^{3}$ Institute of Bioorganic Chemistry, Polish Academy of Sciences, Poznan, Poland, ${ }^{4}$ Faculty of \\ Chemistry, A. Mickiewicz University, Poznan, Poland
}

joanna.loch@uj.edu.pl,loch@chemia.uj.edu.pl

L-asparaginases are used in the treatment of Acute Lymphoblastic Leukemia (ALL). However, the currently utilized bacterial-type enzymes cause several severe side effects. Therefore, there is an urgent need to develop a new generation of therapeutic Lasparaginases. Promising candidates can be found among plant-type L-asparaginases, which function as Ntn-hydrolases. Ntn-hydrolases are produced as inactive precursors and develop enzymatic activity in an autoproteolytic maturation process. Unfortunetely, therapeutic use of wild-type (WT) plant-type enzymes is not possible due to their low substrate affinity (mM). Random mutagenesis is a protein engineering tool that can be used to enhance enzyme efficiency and study structure-function relationship. In this project, locally performed random mutagenesis was used to generate a library of mutants of plant-type L-asparaginase from E.coli (EcAIII). Several new variants of EcAIII were selected from the library and subjected to detailed structural (X-ray crystallography) and biophysical studies (nanoDSF, CD, activity/autoproteolytic tests).
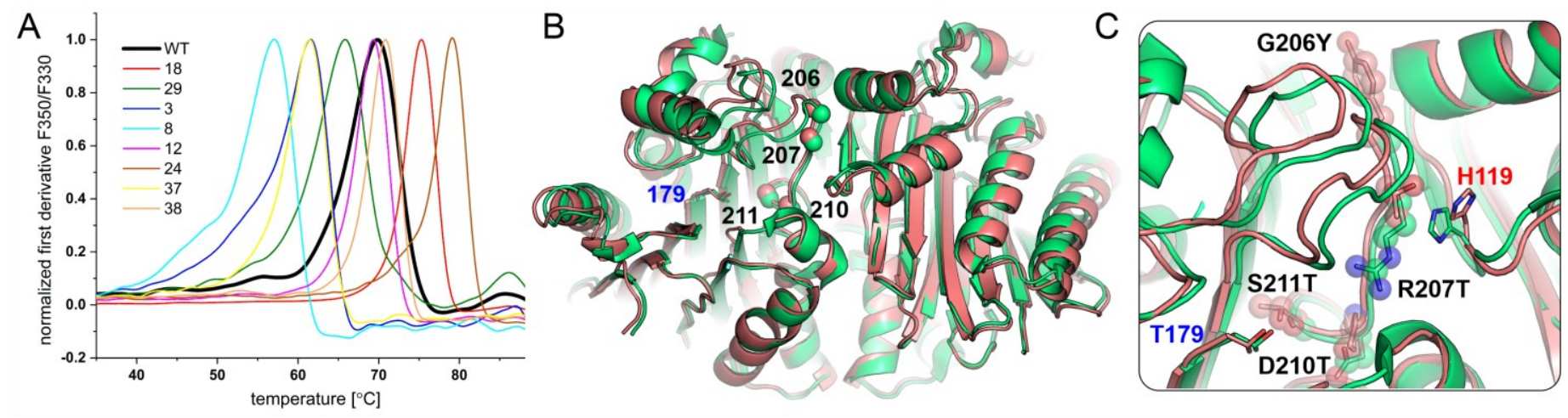

Figure 1. (A) Melting profiles of EcAIII mutants that retained autoprocessing ability (number and line color represents particular mutant from the library). (B) Superposition of WT (green) and EcAIII-8 variant (salmon) with mutation sites and the catalytic T179 marked by their residue numbers. (C) The mutations in EcAIII-8 caused systematic shifts of atoms in the entire protein chain.

The results of our studies revealed that autoproteolysis and enzymatic activity of EcAIII are unrelated events. Some variants of EcAIII retained the ability to autoprocess even in the absence of Arg207 (Fig. 1C), which is critical for catalysis and important for maintaining a H-bond network in the active site. Screening of thermal stability by nanoDSF showed that all analyzed unprocessed EcAIII variants had decreased $T_{m}$ with respect to the WT enzyme. Thermal stability of variants cleaved into the mature $\alpha \beta$ subunits varied, but some mutants with increased $\mathrm{T}_{\mathrm{m}}$ were also found (Fig. 1A). Crystallization experiments proved that it was possible to obtain crystals of all variants cleaved into subunits. This was in stark contrast to the unprocessed mutants, which did not produce any crystals despite of extensive screening. This effect may be related to the presence of a highly disordered $\alpha-\beta$ linker in the uncleaved proteins. CD spectra showed that most of the unprocessed mutants are folded like the WT protein; however, some variants with significant changes in the CD signal were also found. The determined crystal structures (resolution 1.6-2.4 $\AA$ ) showed that the active site of EcAIII is flexible enough to accept different amino acid substitutions; however, the type of substitution affects the H-bond pattern in the active site. Absence of Arg207 affects the overall conformation of the protein and leads to significant shifts of atomic positions in the entire enzyme molecule (Fig. 1B), as illustrated by the C $\alpha$ rmsd value of $1.10 \AA$.

Keywords: plant-type L-asparaginase; random mutagenesis, protein engineering, Ntn-hydrolase, thermal stability

Work supported by National Science Centre (NCN, Poland) grants 2020/38/E/NZ1/00035 and 2019/03/X/NZ1/00584. 